\title{
Trends in cardiovascular admissions and procedures for people with and without diabetes in England, 1996-2005
}

\author{
A. Bottle $\cdot$ C. Millett $\cdot$ K. Khunti $\cdot$ A. Majeed
}

Received: 12 March 2008 / Accepted: 11 September 2008 / Published online: 22 October 2008

(C) Springer-Verlag 2008

\begin{abstract}
Aims/hypothesis The aim of this study was to compare 10 -year trends in admissions, with and without diabetes recorded, for myocardial infarction, angina, stroke, percutaneous coronary interventions (PCI) and coronary artery bypass graft (CABG).

Methods We used national hospital-activity data in England collected between 1996 and 2005 and compared trends in admissions, after adjusting for age, sex and area-level deprivation.

Results Overall, there was a modest fall in the number of admissions for angina and little change in the numbers of admissions for myocardial infarction (MI) and stroke. From $1996 / 1997$ to $2005 / 2006$, the numbers of admissions with diabetes recorded rose for each of MI, angina and stroke; the proportion of admissions with type 2 diabetes recorded rose from $7.2 \%$ to $13.9 \%$ for MI, from $6.7 \%$ to $15.3 \%$ for angina and from $6.2 \%$ to $11.3 \%$ for stroke. Over the 10 -year period, after adjusting for age, sex and deprivation, the number of admissions for $\mathrm{CABG}$ rose about threefold; for PCI, the number of admissions with diabetes recorded rose 15 -fold, compared with a fourfold increase in the number of admissions with diabetes not recorded.

Conclusions/interpretation We found significant increases in the numbers of admissions with type 2 diabetes recorded for major cardiovascular events and procedures, which has important financial and public-health implications. Better prevention of type 2 diabetes in at-risk patients and
\end{abstract}

A. Bottle $(\bowtie) \cdot$ C. Millett $\cdot$ A. Majeed

Department of Primary Care and Social Medicine,

Imperial College London,

3rd Floor, Reynolds Building, Charing Cross Campus,

London W6 8RP, UK

e-mail: robert.bottle@imperial.ac.uk

K. Khunti

Department of Health Sciences, University of Leicester,

Leicester, UK aggressive cardiovascular risk-factor management in current patients with diabetes is needed.

Keywords Cardiovascular $\cdot$ Diabetes $\cdot$ Hospital admissions

\begin{tabular}{|c|c|}
\hline \multicolumn{2}{|c|}{ Abbreviations } \\
\hline CABG & Coronary artery bypass graft \\
\hline HES & $\begin{array}{l}\text { Hospital episode statistics (routine hospital } \\
\text { admissions dataset for England) }\end{array}$ \\
\hline ICD-10 & $\begin{array}{l}\text { International Classification of Diseases, tenth } \\
\text { revision }\end{array}$ \\
\hline MI & Myocardial infarction \\
\hline NHS & National Health Service \\
\hline PCI & Percutaneous coronary intervention \\
\hline
\end{tabular}

Introduction

The incidence and prevalence of both type 1 and type 2 diabetes are increasing worldwide [1]. Macrovascular complications of diabetes, which include stroke, transient ischaemic attacks, myocardial infarction and angina, are responsible for considerable morbidity and mortality for people with diabetes. The risk of cardiovascular events in people with diabetes is two to four times the risk in people without diabetes [2]. The management of these complications places a substantial and growing burden on healthcare systems [3].

There has been a reduction in mortality from cardiovascular disease over the past 30 years in most developed countries $[4,5]$ which can be partly attributed to improved management of risk factors over this period [6,7]. Whilst recent studies from the US have shown reductions in incident cardiovascular disease events among adults with diabetes, these reductions have been much smaller than in people without diabetes $[8,9]$. As the number of new cases of diabetes has increased markedly over recent years [6], 
the absolute burden of cardiovascular disease among people with diabetes is likely to have increased substantially [10].

Trends showing a reduction in cardiovascular mortality have also been reported in the UK [11], and this has generally been attributed to a reduction of cardiovascular risk factors $[12,13]$. However, little is known about recent UK trends in hospitalisations of people with and without diabetes. Hospital admissions account for the majority of the economic cost of cardiovascular disease - estimated at $76 \%$ of the total cost [11]. An evaluation of temporal variation may be useful for future healthcare planning and resource allocation. We investigated trends in major cardiovascular events requiring hospital admission and procedures between 1996 and 2005 using routine admissions data from all National Health Service (NHS; public) hospitals in England, comparing admissions with type 1 or type 2 diabetes recorded and admissions without diabetes recorded.

\section{Methods}

The study was carried out with Section 60 approval from the Patient Information Advisory Group (PIAG) (to hold confidential data and analyse them for research purposes) and approval from St Mary's Local Research Ethics Committee. We examined hospital episode statistics (HES) submitted between the financial years 1996-1997 and 2005-2006 for all NHS hospital trusts in England. The dataset also included private patients treated in NHS hospitals but not in private hospitals. The basic unit of the database was the 'consultant episode', which covered the period during which a patient was under the care of a given consultant (an admission can comprise more than one episode). By matching patient details and admission dates, these were linked into admissions. If the patient was transferred to another hospital, this was not counted as a separate admission.

The International Classification of Diseases, tenth revision (ICD-10) (http://www.who.int/classifications/icd/ en/), and the Office of Population, Censuses and Surveys Classification of Surgical Operations and Procedures, fourth revision (OPCS-4) codes were used to define diagnoses and operations, respectively. HES now contains 14 diagnosis fields, of which the first is the primary, and 12 procedure fields, the first of which corresponds to the most major procedure during the episode. HES has incorporated ICD-10 diagnostic coding since 1995/1996.

Admissions were divided into three groups for analysis: no diabetes, type 1 diabetes (ICD-10 E10, 'IDDM') and type 2 diabetes (ICD-10 E11, 'NIDDM'). Complications of diabetes, considered as the primary diagnosis to be studied with diabetes as the second or subsequent diagnosis, were myocardial infarction (ICD-10 I21-I22), angina (ICD-10 I20) and stroke (ICD-10 I60-I64). Cardiovascular interventions studied were percutaneous coronary intervention (PCI; OCPS-4 K49-K50) and coronary artery bypass graft (CABG; OCPS-4 K40-K46). For inclusion in the dataset, these OCPS- 4 codes could be recorded in any procedure field in any episode during the admission.

Socioeconomic deprivation quintiles were assigned using the Carstairs index of deprivation [14]. Patients' postcodes were mapped to an output area (with an average of 1,500 people), which was then given a deprivation score and assigned to quintiles of equal population nationally.

Admission data for the same patient were determined using a combination of date of birth, sex and postcode as these were the only identifiers available for all years of data. We calculated the number of admissions per admitted patient for each year and the number of different patients admitted each year. To calculate death rates, each patient's final admission in the year was used as the denominator and in-hospital deaths were identified using the method of discharge field for the numerator.

Data analysis was performed using the Statistical Package for the Social Sciences (SPSS) v14 and the R statistical package (http://www.r-project.org/). Poisson regression was used to model the number of admissions per year, adjusting for age, sex, deprivation quintile and diagnosis group. Logistic regression was used for determining trends in mortality rates.

\section{Results}

Over the 10-year study period, there were 834,378 admissions with myocardial infarction $(728,309$ without diabetes; 17,397 with type 1 diabetes; 88,672 with type 2 diabetes), 1,104,170 with angina $(968,045 ; 22,719$; $113,406)$ and 888,408 with stroke $(801,546 ; 11,993$; $74,869)$. There were 383,593 PCIs $(344,661 ; 6,266$; $32,666)$ and 237,504 CABGs $(206,115 ; 6,686 ; 24,703)$. People admitted with type 1 diabetes were younger, especially those admitted for stroke, compared with people admitted without diabetes or with type 2 diabetes (Table 1). People admitted with type 2 diabetes had a mean age similar to those without diabetes. There were generally lower proportions of men in the type 1 groups in 1995/ 1996, though the proportions had risen noticeably by $2005 /$ 2006. A higher proportion of admissions of people with type 1 and type 2 diabetes recorded were assigned to areas in the most deprived quintile compared with those admitted without diabetes recorded.

Of all admissions for myocardial infarction in 1996/ $1997,7.2 \%$ had type 2 diabetes recorded, rising to $13.9 \%$ in 2005/2006. Similarly, the proportion with type 2 diabetes 
Table 1 Demographic data from 1996/1997 and 2005/2006 for admissions for MI, angina, stroke, PCI and CABG

\begin{tabular}{|c|c|c|c|c|c|c|}
\hline \multirow[t]{2}{*}{ Condition } & \multicolumn{3}{|l|}{$1996 / 1997$} & \multicolumn{3}{|l|}{$2005 / 2006$} \\
\hline & No diabetes & Type 1 & Type 2 & No diabetes & Type 1 & Type 2 \\
\hline \multicolumn{7}{|l|}{ MI } \\
\hline Number of admissions & 74,225 & 1,515 & 5,861 & 78,604 & 1,160 & 12,824 \\
\hline $\begin{array}{l}\text { Number of patients (ratio of admissions } \\
\text { to admitted patients) }\end{array}$ & $67,953(1.09)$ & $1,384(1.09)$ & $5,382(1.09)$ & $60,600(1.30)$ & $930(1.25)$ & $10,279(1.25)$ \\
\hline Age, years (IQR) & $69.8(60-78)$ & $67.2(60-75)$ & $69.6(62-78)$ & $69.5(59-80)$ & $66.7(57-77)$ & $71.7(64-81)$ \\
\hline Sex: proportion of men $(\%)$ & 64.3 & 54.0 & 60.9 & 64.9 & 62.2 & 61.2 \\
\hline Proportion in most deprived quintile (\%) & 21.9 & 24.8 & 26.5 & 18.8 & 24.6 & 26.2 \\
\hline \multicolumn{7}{|l|}{ Angina } \\
\hline Number of admissions & 97,023 & 2,025 & 7,095 & 83,434 & 1,387 & 15,302 \\
\hline $\begin{array}{l}\text { Number of patients (ratio of admissions } \\
\text { to admitted patients) }\end{array}$ & $77,409(1.25)$ & $1,605(1.26)$ & $5,604(1.27)$ & $68,363(1.22)$ & $1,096(1.27)$ & $12,382(1.24)$ \\
\hline Age, years (IQR) & $66.3(57-75)$ & $64.1(57-72)$ & $67.5(60-75)$ & $67.4(58-78)$ & $64.2(56-73)$ & $68.2(60-77)$ \\
\hline Sex: proportion of men $(\%)$ & 60.3 & 53.3 & 60.4 & 57.7 & 58.7 & 60.1 \\
\hline Proportion in most deprived quintile (\%) & 25.7 & 30.1 & 32.3 & 23.3 & 32.9 & 31.5 \\
\hline \multicolumn{7}{|l|}{ Stroke } \\
\hline Number of admissions & 77,005 & 1,212 & 5,130 & 79,446 & 781 & 10,221 \\
\hline $\begin{array}{l}\text { Number of patients (ratio of admissions } \\
\text { to admitted patients) }\end{array}$ & $69,385(1.11)$ & $1,126(1.08)$ & $4,811(1.07)$ & $68,714(1.16)$ & $696(1.12)$ & $9,149(1.12)$ \\
\hline Age, years (IQR) & $80.6(67-83)$ & $69.3(62-78)$ & $73.9(67-82)$ & $83.9(66-84)$ & $69.1(61-79)$ & $74.7(68-82)$ \\
\hline Sex: proportion of men $(\%)$ & 45.8 & 49.3 & 51.1 & 46.6 & 55.6 & 51.9 \\
\hline Proportion in most deprived quintile $(\%)$ & 22.2 & 26.0 & 26.7 & 19.1 & 24.7 & 24.9 \\
\hline \multicolumn{7}{|l|}{ PCI } \\
\hline Number of procedures & 10,574 & 126 & 461 & 34,730 & 607 & 5,268 \\
\hline $\begin{array}{l}\text { Number of patients (ratio of procedures } \\
\text { to admitted patients) }\end{array}$ & $9,927(1.07)$ & $111(1.14)$ & $432(1.07)$ & $33,290(1.04)$ & $557(1.09)$ & $4,928(1.07)$ \\
\hline Age, years (IQR) & $61.5(53-68)$ & $59.3(52-67)$ & $63.1(57-70)$ & $63.9(56-72)$ & $61.8(54-71)$ & $64.6(58-72)$ \\
\hline Sex: proportion of men $(\%)$ & 73.8 & 58.3 & 70.3 & 74.1 & 67.7 & 69.9 \\
\hline Proportion in most deprived quintile (\%) & 20.5 & 23.6 & 25.8 & 19.3 & 27.8 & 30.0 \\
\hline \multicolumn{7}{|l|}{ CABG } \\
\hline Number of procedures & 17,155 & 312 & 1,182 & 16,175 & 523 & 3,168 \\
\hline $\begin{array}{l}\text { Number of patients (ratio of procedures } \\
\text { to admitted patients) }\end{array}$ & $17,082(1.00)$ & $308(1.01)$ & $1,175(1.01)$ & $16,108(1.00)$ & $523(1.00)$ & $3,145(1.01)$ \\
\hline Age, years (IQR) & $63.3(57-69)$ & $60.3(55-67)$ & $62.8(58-69)$ & $67.6(60-74)$ & $63.4(57-71)$ & $66.5(61-73)$ \\
\hline Sex: proportion of men $(\%)$ & 79.0 & 68.7 & 77.7 & 78.9 & 72.3 & 76.4 \\
\hline Proportion in most deprived quintile (\%) & 19.5 & 24.5 & 24.7 & 16.2 & 23.6 & 23.3 \\
\hline
\end{tabular}

IQR interquartile range

admitted for angina increased from $6.7 \%$ in $1996 / 1997$ to $15.3 \%$ in $2005 / 2006$, and for stroke the proportion increased from $6.2 \%$ to $11.3 \%$ over the same period. By contrast, the proportion of all patients admitted with type 1 diabetes recorded showed a general downward trend except for a slight increase at the start of the period. Figure 1 shows the number of admissions for myocardial infarction, angina, stroke, percutaneous coronary interventions and coronary artery bypass grafting in the years 1995/1996$2005 / 2006$ by recorded diabetes status. Admissions per year are shown relative to 1996 totals and on a log scale so that upward and downward trends are given equal importance (relative risks are not symmetrical about 1 ).

There was nearly a fourfold increase in total PCIs over the 10 year study period. People with type 2 diabetes accounted for $4.6 \%$ of these procedures in $1996 / 1997$, rising to $11.8 \%$ in $2005 / 2006$. Total CABG operation numbers remained static, but the percentage accounted for by people with type 2 diabetes increased from $6.2 \%$ in $1996 / 1997$ to $15.8 \%$ in $2005 / 2006$. The numbers of patients with type 1 diabetes undergoing $\mathrm{CABG}$ showed only a modest increase between 1996/1997 and 2005/2006.

After adjusting for age, sex and deprivation quintile, a small but significant trend showing a modest decline in the number of cardiovascular events without diabetes recorded was seen, with a rise in the number of PCIs performed and slight reduction in the number of $\mathrm{CABG}$ operations performed (Table 2). The number of admissions with type 1 diabetes recorded showed a similar trend, but with a nonsignificant increase in the number of $\mathrm{CABG}$ operations and 


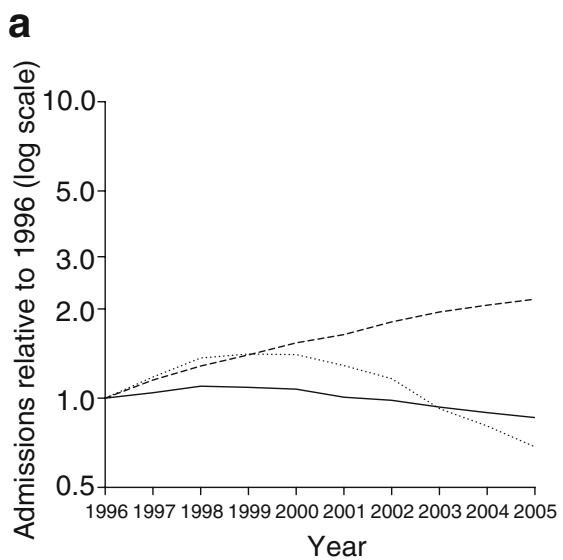

\section{b}
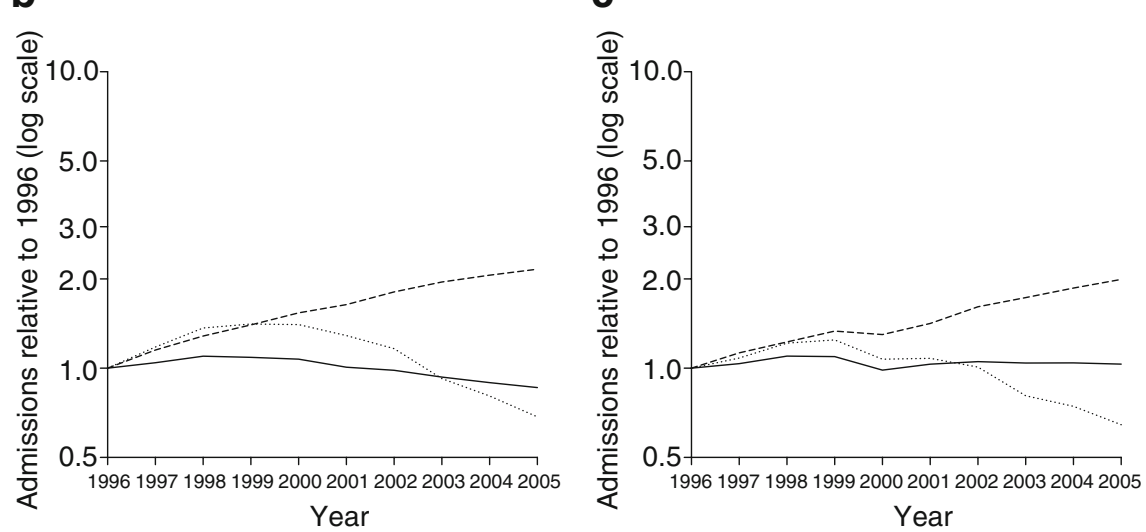

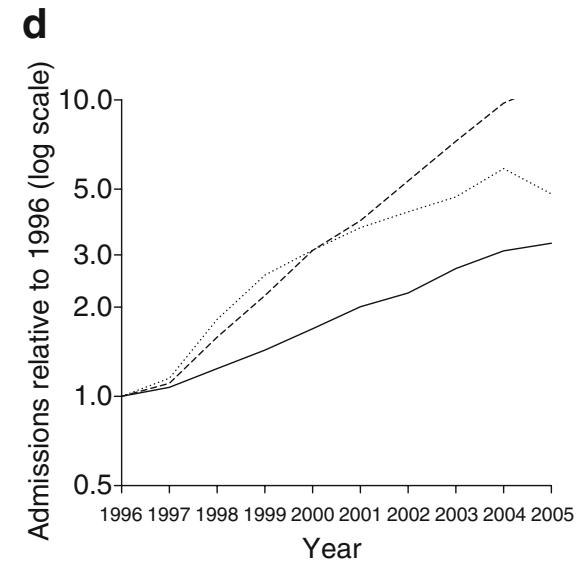

Fig. 1 Hospital admissions and procedures by diagnosis in the period 1995/1996 to $2005 / 2006$ relative to 1995/1996: (a) myocardial infarction, (b) angina, (c) stroke, (d) percutaneous coronary inter-

a smaller increase in PCIs relative to those without diabetes. Trends for type 1 diabetes were not as well described by a simple linear pattern as trends for the other two groups (i.e. no diabetes recorded and type 2 diabetes recorded). By contrast, the proportions of all patients with cardiovascular events and procedures who had type 2 diabetes recorded showed significant increases, particularly for PCI where there was a $30 \%$ increase per annum $(p<0.001)$. The estimates were little changed by counting admitted patients rather than number of admissions (values in brackets in Table 2).

There has been a trend of increasing admissions per admitted patient for MI for all three groups, from 1.09 in 1996/1997 to 1.25 for people with diabetes and 1.30 for people without diabetes in 2005/2006. The rise for stroke, from 1.07 to 1.12 for people with type 2 diabetes and from 1.11 to 1.16 for people without diabetes, was much more modest; angina showed a slight reduction in admissions. Ratios for CABG showed no change (1.00 or 1.01 throughout) and for PCIs there was a slight reduction from 1.07 to 1.04 for people without diabetes and non-significant decreases for people with type 1 or type 2 diabetes.

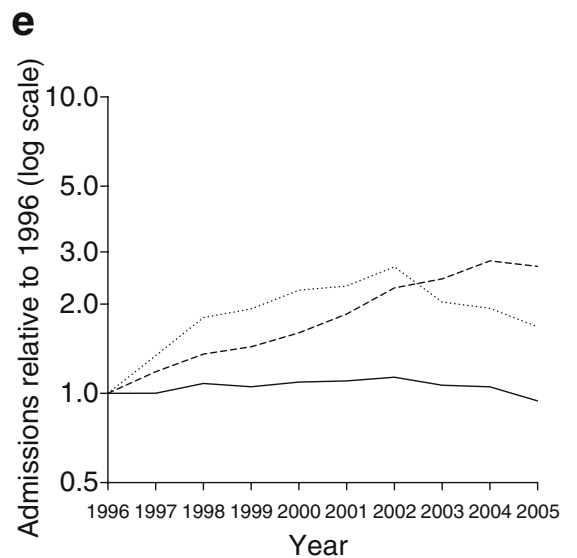

ventions, (e) coronary artery bypass graft. Continuous line, no diabetes; dotted line, type 1 diabetes; dashed line, type 2 diabetes

Table 3 shows the odds ratios for mortality in people with type 1 and type 2 by event or procedure relative to people without diabetes in 1996/1997 and 2005/2006 (the denominators are numbers of patients admitted in each year). Death rates fell for all three groups during the 10year period for MI, stroke and CABG. The odds ratios were higher for people with type 1 and type 2 diabetes for MI and angina at both ends of the study period, and for stroke and PCI in 2005/2006. The most noticeable change between 1996/1997 and 2005/2006 was for people with type 1 diabetes admitted for angina, with a doubling in the odds ratio. In general, however, there was little difference in the death rates for people with and without diabetes at the start compared with the end of the study period.

\section{Conclusion}

The findings of this national study have important publichealth consequences. We found different trends over the last 10 years in admissions with and without type 2 diabetes 
Table 2 Change in numbers of admissions/procedures per year, adjusted for age, sex and quintile of deprivation relative to 1996/1997 numbers

\begin{tabular}{|c|c|c|c|c|c|}
\hline & \multicolumn{5}{|l|}{ Condition } \\
\hline & MI & Angina & Stroke & PCI & CABG \\
\hline \multicolumn{6}{|l|}{ No diabetes } \\
\hline Change per year relative to $1996 / 1997$ & $1.007(0.989)$ & $0.979(0.982)$ & $0.999(0.996)$ & $1.150(1.154)$ & $0.994(1.000)$ \\
\hline $95 \% \mathrm{CI}$ & $1.001-1.014$ & $0.973-0.984$ & $0.995-1.005$ & $1.142-1.159$ & $0.989-0.999$ \\
\hline Change in 10 years relative to $1996 / 1997$ & $1.08(0.90)$ & $0.81(0.83)$ & $1.00(0.96)$ & $4.06(4.20)$ & $0.94(1.00)$ \\
\hline$p$ value & 0.04 & $<0.001$ & 0.92 & $<0.001$ & 0.035 \\
\hline \multicolumn{6}{|l|}{ Type 1 diabetes } \\
\hline Change per year relative to $1996 / 1997$ & $0.969(0.957)$ & $0.954(0.958)$ & $0.951(0.949)$ & $1.133(1.150)$ & $1.030(1.041)$ \\
\hline $95 \% \mathrm{CI}$ & $0.962-0.978$ & $0.947-0.963$ & $0.944-0.960$ & $1.118-1.148$ & $1.020-1.041$ \\
\hline Change in 10 years relative to $1996 / 1997$ & $0.73(0.65)$ & $0.63(0.65)$ & $0.61(0.59)$ & $3.48(4.06)$ & $1.35(1.50)$ \\
\hline$p$ value & $<0.001$ & $<0.001$ & $<0.001$ & $<0.001$ & $<0.001$ \\
\hline \multicolumn{6}{|l|}{ Type 2 diabetes } \\
\hline Change per year relative to $1996 / 1997$ & $1.099(1.082)$ & $1.085(1.090)$ & $1.076(1.071)$ & $1.309(1.316)$ & $1.115(1.120)$ \\
\hline $95 \% \mathrm{CI}$ & $1.092-1.107$ & $1.079-1.091$ & $1.07-1.082$ & $1.298-1.321$ & $1.107-1.123$ \\
\hline Change in 10 years relative to $1996 / 1997$ & $2.58(2.21)$ & $2.26(2.36)$ & $2.08(1.99)$ & $14.76(15.64)$ & $2.96(3.10)$ \\
\hline$p$ value & $<0.001$ & $<0.001$ & $<0.001$ & $<0.001$ & $<0.001$ \\
\hline
\end{tabular}

Values are change in numbers of admissions/procedures with change in numbers of admitted patients in parentheses

The change in 10 years is derived by raising the change per year to the tenth power and assumes a linear trend; this assumption is not well met for type 1 diabetes

recorded. Admissions with type 1 diabetes showed similar trends to admissions without diabetes. Whereas the numbers of admissions without diabetes have shown little change or slight falls in myocardial infarction, angina, stroke and CABG procedures, the numbers of admissions with type 2 diabetes have seen yearly rises of between $8 \%$ and $12 \%$. This is due partly to people being admitted more often: all three groups have seen rises of approximately $15 \%$ over the 10 years in the number of admissions per admitted patient for myocardial infarction. We also found a significant increase in numbers of PCI procedures in all patients; however, the trend is substantially greater with a $30 \%$ annual increase in PCI procedures in those with type 2 diabetes recorded. The decline in mortality in people with diabetes may explain some of the increased numbers of admissions for cardiovascular disease and procedures with type 2 diabetes recorded. This is likely to be a major factor contributing to the substantial increase in the proportion of admissions with cardiovascular disease in our study.

We found a reduction in the number of hospital admissions from around 1999 to 2000 for people with type 1 diabetes. This decline may have been due to the introduction of a new diagnostic classification of diabetes in 1999 when the terms type 1 and type 2 diabetes were re-introduced [15]. It may also reflect a smaller increase in the prevalence of type 1 diabetes compared with type 2 diabetes.

The mortality rate and rates of stroke and further infarcts following a myocardial infarction fell in many countries between 1999 and 2006 [8]. We also found that the mortality rates following MI and stroke decreased over the 10 years of this study. Data from the Health Survey for England (http://www.ic.nhs.uk/statistics-and-data-collections/healthand-lifestyles/health-survey-for-england/health-survey-forengland-2004-updating-of-trend-tables-to-include-2004-data) show an increase in overall diabetes prevalence from $2.9 \%$ in 1998 to $4.3 \%$ in 2003 for men and rises from $1.9 \%$ to $3.4 \%$ in women over the same period. If we assume a linear trend, then the estimated rise between 1995/1996 and 2005/ 2006 is from $2.3 \%$ to $4.9 \%$ in men (a rate ratio of 2.1 ) and from $1.6 \%$ to $4.0 \%$ in women (a rate ratio of 2.5 ). By comparison, the numbers of admissions for the three diagnoses examined in our study increased by between 2.0 and 2.2 times; revascularisations increased by 2.6 times for $\mathrm{CABG}$ and 8.6 times for PCI.

Increasing PCI numbers, seen in all groups, reflect the wider availability of this procedure and the trend for earlier intervention in patients with coronary heart disease. Earlier diagnosis of coronary heart disease coupled with more advanced medical management and greater use of PCI helps to explain the fall of CABG surgery in the nondiabetic population. The very large increase in PCI numbers with type 2 diabetes recorded probably reflects the more advanced atherosclerotic coronary disease profile of these patients. People with diabetes undergoing PCIs are known to have significantly more disease progression and are more likely to experience the need for repeated revascularisation [16]. While PCI use has increased in people without diabetes, the number of $\mathrm{CABG}$ operations performed has risen greatly in people with type 2 diabetes. The disparity between the numbers of people undergoing $\mathrm{CABG}$ with 
Table 3 Deaths and odds ratios by diabetes status for admitted patients (rather than total admissions) in 1996/1997 and 2005/2006, adjusted for age, sex and quintile of deprivation

\begin{tabular}{|c|c|c|c|c|c|c|c|c|}
\hline \multirow[t]{2}{*}{ Diabetes status } & \multicolumn{4}{|l|}{ 1996/1997 } & \multicolumn{4}{|c|}{$2005 / 2006$} \\
\hline & Deaths (n) & Death rate $(\%)$ & OR $(95 \% \mathrm{CI})$ & $p$ value & Deaths & Death rate $(\%)$ & OR $(95 \% \mathrm{CI})$ & $p$ value \\
\hline \multicolumn{9}{|l|}{ MI } \\
\hline No diabetes & 12,926 & 19.0 & 1 & & 8,297 & 13.7 & 1 & \\
\hline Type 1 DM & 354 & 25.6 & $1.69(1.49-1.93)$ & $<0.001$ & 163 & 17.5 & $1.72(1.44-2.06)$ & $<0.001$ \\
\hline Type 2 DM & 1,183 & 22.0 & $1.17(1.09-1.26)$ & $<0.001$ & 1,652 & 16.1 & $1.13(1.06-1.20)$ & $<0.001$ \\
\hline \multicolumn{9}{|l|}{ Angina } \\
\hline No diabetes & 1,031 & 1.3 & 1 & & 945 & 1.4 & 1 & \\
\hline Type 1 DM & 20 & 1.3 & $1.15(0.74-1.80)$ & 0.534 & 23 & 2.1 & $2.28(1.49-3.48)$ & $<0.001$ \\
\hline Type 2 DM & 104 & 1.9 & $1.37(1.11-1.68)$ & 0.003 & 207 & 1.7 & $1.28(1.10-1.49)$ & 0.002 \\
\hline \multicolumn{9}{|l|}{ Stroke } \\
\hline No diabetes & 25,683 & 37.0 & 1 & & 19,028 & 27.7 & 1 & \\
\hline Type 1 DM & 382 & 33.9 & $1.09(0.96-1.24)$ & 0.194 & 177 & 25.4 & $1.16(0.97-1.39)$ & 0.095 \\
\hline Type 2 DM & 1,697 & 35.3 & $0.97(0.92-1.04)$ & 0.424 & 2,588 & 28.3 & $1.10(1.04-1.15)$ & $<0.001$ \\
\hline \multicolumn{9}{|l|}{ PCI } \\
\hline No diabetes & 103 & 1.0 & 1 & & 195 & 0.6 & 1 & \\
\hline Type 1 DM & 2 & 1.8 & $2.08(0.50-8.63)$ & 0.313 & 7 & 1.3 & $2.29(1.07-4.91)$ & 0.034 \\
\hline Type 2 DM & 4 & 0.9 & $0.75(0.27-2.06)$ & 0.575 & 44 & 0.9 & $1.42(1.02-1.97)$ & 0.040 \\
\hline \multicolumn{9}{|l|}{ CABG } \\
\hline No diabetes & 577 & 3.4 & 1 & & 383 & 2.4 & 1 & \\
\hline Type 1 DM & 11 & 3.6 & $1.15(0.62-2.12)$ & 0.656 & 16 & 3.1 & $1.52(0.91-2.54)$ & 0.110 \\
\hline Type 2 DM & 33 & 2.8 & $0.83(0.58-1.19)$ & 0.307 & 64 & 2.0 & $0.89(0.68-1.16)$ & 0.379 \\
\hline
\end{tabular}

All in-hospital deaths are shown for the diagnosis groups and in-hospital deaths within 30 days of the procedure are shown for PCI and CABG Death-rate denominators are numbers of patients admitted in the year, not total admissions

$D M$ diabetes mellitus

and without diabetes may be because people with diabetes are more likely to have left-main-stem or triple-vessel coronary disease, both of which are indications for CABG surgery $[17,18]$.

Although the use of preventative measures in people at risk of cardiovascular disease has increased substantially over recent years, these measures are not always used in people at high risk [19]. The importance of diabetes as a major cardiovascular-disease risk factor has, however, become more widely known and this may have led to an increased recording of diabetes in hospital episodes. This, in turn, would have contributed to the increase in the proportion of cardiovascular events in patients coded as having diabetes. In this study we have assumed that the changes in admissions by diabetes status are not simply a reflection of temporal changes in diagnostic coding. Although criticisms have been levelled at HES data in the past because of the perceived inaccuracy of coding [20,21], a systematic review of discharge coding rated its accuracy as high, particularly for operations and procedures [22]. With the more common diagnoses and procedures, coding inaccuracies are likely to be minimised [23]. It is also possible that some patients have been misclassified as having type 1 rather than type 2 diabetes prior to introduction of the new classification of diabetes. Such 'diagnostic transfer', which can be due to clinical or administrative reasons, is unlikely to have had a significant impact on the results of this study.

With the advent of troponin testing, the diagnosis of myocardial infarction changed during the study period. While this may have caused an increase in diagnosed events, particularly in people with diabetes because of the higher risk of 'silent' myocardial infarctions, it is unlikely to fully explain the differences seen. Limitations also surround the diagnosis of 'angina'. Unfortunately, data on ethnic differences are missing because ethnicity is poorly coded (missing in approximately $30-40 \%$ of HES data).

We found significant upward trends for type 2 diabetes in the absolute numbers and proportion of the total number of admissions for cardiovascular events. To reduce the burden of disease there is a need for prevention of type 2 diabetes in high-risk groups and aggressive cardiovascularrisk-factor management in patients with existing type 2 diabetes.

Duality of interest A. Bottle is employed within the Dr Foster Unit at Imperial College London. The Unit is funded by a grant from $\mathrm{Dr}$ Foster Intelligence, an independent health service research organisation. The Department of Primary Care and Social Medicine receives support from the National Institute for Health Research Biomedical Research Centre funding scheme and the National Institute of Health Research Collaboration for Leadership in Applied Health Research \& Care programme. The authors declare that there is no duality of interest associated with this manuscript. 


\section{References}

1. Lipscombe LL, Hux JE (2007) Trends in diabetes prevalence, incidence, and mortality in Ontario, Canada 1995-2005: a population-based study. Lancet 369:750-756

2. Kannel WB, McGee DL (1979) Diabetes and cardiovascular disease. The Framingham study. JAMA 241:2035-2038

3. Caro JJ, Ward AJ, O'Brien JA (2002) Lifetime costs of complications resulting from type 2 diabetes in the U.S. Diabetes Care 25:476-481

4. Gillum RF (1994) Trends in acute myocardial infarction and coronary heart disease death in the United States. J Am Coll Cardiol 23:1273-1277

5. Gregg EW, Gu Q, Cheng YJ, Narayan KM, Cowie CC (2007) Mortality trends in men and women with diabetes, 1971 to 2000. Ann Intern Med 147:149-155

6. Imperatore G, Cadwell BL, Geiss L et al (2004) Thirty-year trends in cardiovascular risk factor levels among US adults with diabetes: National Health and Nutrition Examination Surveys, 1971-2000. Am J Epidemiol 160:531-539

7. Saaddine JB, Cadwell B, Gregg EW et al (2006) Improvements in diabetes processes of care and intermediate outcomes: United States, 1988-2002. Ann Intern Med 144:465-474

8. Fox CS, Coady S, Sorlie PD et al (2004) Trends in cardiovascular complications of diabetes. JAMA 292:2495-2499

9. Gu K, Cowie CC, Harris MI (1999) Diabetes and decline in heart disease mortality in US adults. JAMA 281:1291-1297

10. Booth GL, Kapral MK, Fung K, Tu JV (2006) Recent trends in cardiovascular complications among men and women with and without diabetes. Diabetes Care 29:32-37

11. Alexander S, Peto P, Scarborough P et al (2006). Coronary Heart Disease Statistics Fact Sheet 2006. Available from http://www.heartstats. org/, accessed 24 September 2008. London, British Heart Foundation

12. Sytkowski PA, Kannel WB, D'Agostino RB (1990) Changes in risk factors and the decline in mortality from cardiovascular disease. The Framingham Heart Study. N Engl J Med 322:1635-1641
13. Unal B, Critchley JA, Capewell S (2004) Explaining the decline in coronary heart disease mortality in England and Wales between 1981 and 2000. Circulation 109:1101-1107

14. Carstairs V, Morris G (1991) Deprivation and health in Scotland. Aberdeen University Press, Aberdeen

15. Alberti KG, Zimmet PZ (1998) Definition, diagnosis and classification of diabetes mellitus and its complications. Diagnosis and classification of diabetes mellitus: provisional report of a WHO consultation. Diabet Med 15:539-553

16. Natali A, Vichi S, Landi P, Severi S, L'Abbate A, Ferrannini E (2000) Coronary atherosclerosis in type II diabetes: angiographic findings and clinical outcome. Diabetologia 43:632-641

17. Waller BF, Palumbo PJ, Lie JT, Roberts WC (1980) Status of the coronary arteries at necropsy in diabetes mellitus with onset after age 30 years. Analysis of 229 diabetic patients with and without clinical evidence of coronary heart disease and comparison to 183 control subjects. Am J Med 69:498-506

18. Khunti K, Gadsby R, Millett C, Majeed A, Davies MJ (2007) Quality of diabetes care in the UK: comparison of published quality of care reports with results of the Quality and Outcomes Framework for Diabetes. Diabet Med 24:1436-1441

19. Persell SD, Baker DW (2004) Aspirin use among adults with diabetes: recent trends and emerging sex disparities. Arch Intern Med 164:2492-2499

20. McKee M (1993) Routine data: a resource for clinical audit? Qual Health Care 2:104-111

21. Leslie PJ, Patrick AW, Hepburn DA, Scougal IJ, Frier BM (1992) Hospital inpatient statistics underestimate the morbidity associated with diabetes mellitus. Diabet Med 9:379-385

22. Campbell SE, Campbell MK, Grimshaw JM, Walker AE (2001) A systematic review of discharge coding accuracy. J Public Health Med 23:205-211

23. Dixon J, Sanderson C, Elliott P, Walls P, Jones J, Petticrew M (1998) Assessment of the reproducibility of clinical coding in routinely collected hospital activity data: a study of two hospitals. J Pub Health Med 20:63-69 